\title{
Glucose-sensitive silicone hydrogel contact lens toward tear glucose monitoring
}

Ramachandram Badugu

Edward Albert Reece

Joseph R. Lakowicz 


\title{
Glucose-sensitive silicone hydrogel contact lens toward tear glucose monitoring
}

\author{
Ramachandram Badugu, ${ }^{\mathrm{a}, *}$ Edward Albert Reece, ${ }^{\mathrm{b}, \mathrm{c}}$ and Joseph R. Lakowicz ${ }^{\mathrm{a}}$ \\ aUniversity of Maryland School of Medicine, Center for Fluorescence Spectroscopy, Department of Biochemistry and Molecular Biology, \\ Baltimore, Maryland, United States \\ bUniversity of Maryland School of Medicine, Department of Obstetrics, Gynecology and Reproductive Sciences, Baltimore, Maryland, \\ United States \\ 'University of Maryland School of Medicine, Department of Biochemistry and Molecular Biology, Baltimore, Maryland, United States
}

\begin{abstract}
Accurate and reliable monitoring of blood glucose is needed for the treatment of diabetes, which has many challenges, including lack of patient compliance. Measuring tear glucose is an alternative to traditional finger-stick tests used to track blood sugar levels, but glucose sensing using tears has yet to be achieved. We report a methodology for possible tear glucose monitoring using glucose-sensitive silicone hydrogel (SiHG) contact lenses, the primary type of lenses available in today's market. Initially, we assessed the interpenetrating polymer network, with nearly pure silicone and water regions, existing in the SiHGs using a polarity-sensitive probe Prodan. We then synthesized a glucose-sensitive fluorophore Quin-C18 with a hydrophobic side chain for localization of probe at the interfacial region. Using our glucose-sensing contact lens, we were able to measure varying concentrations of glucose in an in-vitro system. The Quin-C18 strongly bound to the lenses with insignificant leaching even after multiple rinses. The lenses displayed a similar response to glucose after three months of storage in water. This study demonstrates that it may be possible to develop a contact lens for continuous glucose monitoring in the near term, using our concept of fluorophore binding at the siliconewater interface. () 2018 Society of Photo-Optical Instrumentation Engineers (SPIE) [DOI: 10.1117/1.JBO.23.5.057005]
\end{abstract}

Keywords: diabetes mellitus; glucose monitoring; tear glucose; fluorescence spectroscopy and sensing; glucose sensors; contact lens.

Paper 170749RR received Nov. 21, 2017; accepted for publication Apr. 13, 2018; published online May 17, 2018.

\section{Introduction}

Diabetes mellitus is a chronic disorder affecting more than 27 million individuals in the United States and over 442 million people worldwide as of 2014. ${ }^{1}$ Without further intervention, these numbers are expected to increase to 642 million by the year $2040 .^{2}$ Diabetes mellitus commonly manifests as an insulin- (type 1) or noninsulin-dependent (type 2) form and a pregnancy-specific (gestational) form. ${ }^{3}$ The long-term consequences of elevated blood glucose include neuropathies, retinopathies, cardiovascular diseases, blindness, and a higher risk of birth defects in infants born to women with diabetes in pregnancy. ${ }^{4-9}$

Successful management of diabetes depends on strict glycemic monitoring, which is aided by routine and accurate glucose monitoring by both healthcare practitioners and the patient. Despite clinical evidence supporting the importance of blood glucose self-testing (BGST), patient compliance with routine testing remains an issue. At present, several methods are used for continuous glucose monitoring. ${ }^{10,11}$ The latter can be performed noninvasively, ${ }^{12-15}$ minimally invasively, i.e., with implantable pellets, ${ }^{16-18}$ using microneedle glucose sensors, ${ }^{19}$ or invasively using intravenous glucose electrodes. ${ }^{20}$ Implanted electrodes are used to control insulin pumps, but there can be a site of infection that needs to be replaced at regular intervals. These implanted sensors also do not provide stable readings, and

*Address all correspondence to: Ramachandram Badugu, E-mail: rbadugu@ som.umaryland.edu recalibration is recommended with a finger stick using fresh blood four times per day, resulting in only an incremental health benefit. $^{21,22}$ The inconvenience, discomfort, and expense of frequent finger stick or blood sample testing all play a role in a patients' compliancy to BGST.

The need for more convenient and acceptable blood glucose estimation methods resulted in extensive research to develop various approaches and to obtain continuous measurements of blood glucose. The basic mechanism of glucose sensing has not changed for over 40 years. The majority of self-testing glucometers are based on glucose oxidase and colorimetric or electrochemical detection of the hydrogen peroxide $\left(\mathrm{H}_{2} \mathrm{O}_{2}\right)$. The signal drift is thought to be due to several reasons, including damage of glucose oxidase by the $\mathrm{H}_{2} \mathrm{O}_{2}$, depletion of oxygen around the sensor, and formation of a tissue capsule altering glucose diffusion to the electrode. ${ }^{23}$ Accordingly, the decrease or drift in sensor response in implanted sensors or pellets results in the need for frequent calibration with a fresh blood sample. Other methods were also tested, including optical rotation, photonic crystals, Raman scattering, and impedance and NIR spectroscopy. ${ }^{24-29}$ There have also been multiple attempts for semi-invasive glucose measurements of interstitial fluid obtained using microneedles, suction, and reverse iontophoresis as in the GlucoWatch. ${ }^{30-32}$

The failures of measurements of glucose in blood or interstitial fluid and the difficulties of obtaining frequent fresh

$1083-3668 / 2018 / \$ 25.00$ @ 2018 SPIE 


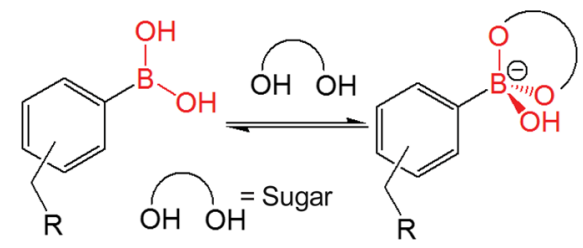

Fig. 1 Simplified scheme for boronic acid binding to sugars.
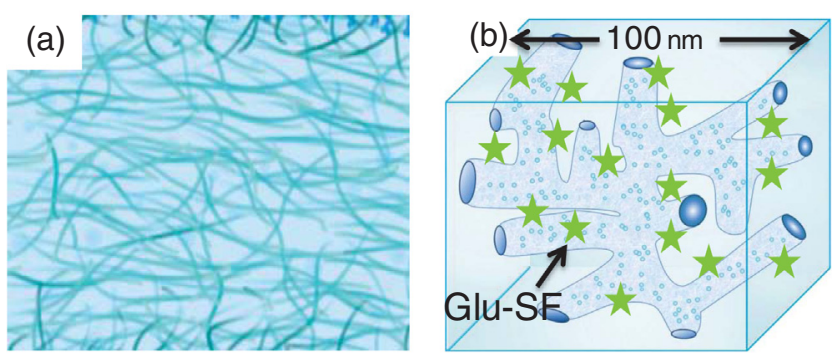

Fig. 2 (a) Schematic cross section of HG contact lens showing the homogeneous interior structure. (b) Schematic of a small region of a SiHG showing the hydrophylic and hydrophobic IPNs. The dimension is an approximation.

blood samples have resulted in the search for alternative sites or fluids to determine glucose concentrations. ${ }^{33-37}$ There is reasonable evidence to indicate that tear glucose follows blood glucose concentrations with $\sim 5$ - min time delay. ${ }^{33-37}$ However, tear glucose measurements have not been widely used because of sample collection issues. Any direct contact with the eye results in changes in the rate of tear production and, thus, loss of the correlation between blood glucose and tear glucose. Collection methods that caused the least eye irritation showed the best correlation between tears and blood glucose. ${ }^{33-37}$ In this regard, using contact lens as a sampling/diagnosis format is a superior method with unperturbed correlation between blood glucose and tear glucose.

Measurements of tear glucose levels have recently been investigated using GlucoLens, being developed by Google $X^{38,39}$ The GlucoLens contains the electronic components and glucose sensors to allow continuous glucose measurements. However, the need for complex and embedded electronics may prevent the GlucoLens from becoming a daily use product. For safety reasons and patient choice, the daily use contact lens is the dominant mode of use in developed countries. ${ }^{40-42}$ On the other hand, continuous monitoring of glucose levels in tears can be greatly simplified using glucose-sensitive fluorophores (Glu-SFs) embedded into a contact lens. Glu-SFs are almost always based on complexation of glucose with a boronic acid moiety (Fig. 1). A fluorescent glucose-sensitive contact lens (Glu-CL) would use remote optical detection to avoid sensor contact with the eyes. The limiting factor for creating a Glu-CL is the lack of Glu-SFs, which respond to tear glucose concentrations when placed into a contact lens. Many known Glu-SFs, which work in aqueous buffer solutions, ${ }^{36,37,43-46}$ show little or no response in nonsilicone hydrogel (HG) lenses. ${ }^{36,37}$ Over the period of time, the contact lens chemistry has developed drastically. The previous generation of contact lenses and some currently used lenses are made from cross-linked polymers of hydroxyethylmethacrylate (HEMA) and similar monomers, which form a network of interwoven polymer strands [Fig. 2(a)]. The spatial distribution of these interiors is uniform throughout (a)

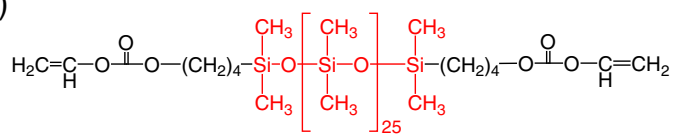

(b)

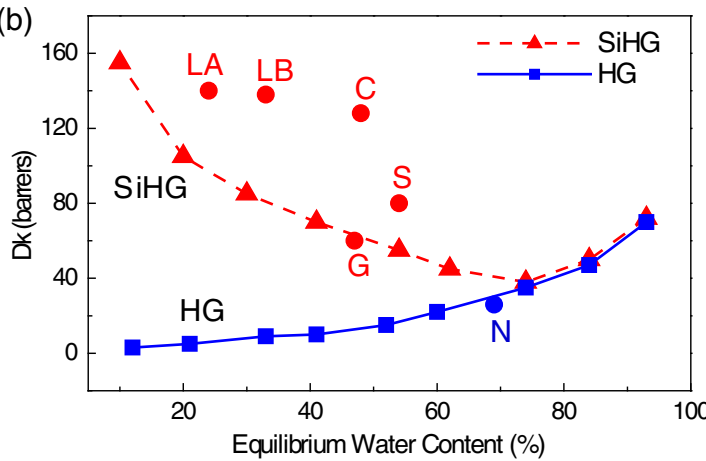

Fig. 3 (a) Typical monomer used in a silicone HG contact lens. (b) Water content and $D k$ values of $H G$ and $\mathrm{SiHG}$ lenses. $L A$, Lotrafilcon A; LB, Lotrafilcon B; G, Galyfilcon A; C, Comfilcon A; $S$, Stenfilcon A; and N, Nelficon A. Comfilcon A is a third-generation SiHG with improved Dk and high water content.

the lens, and in some cases, oxygen transport from the air limits the duration a contact lens can be continuously worn. Oxygen transport in HEMA-type lenses can be increased with higher water content (Fig. 3). However, the lenses with high water content become too frail for practical use, which includes removal, insertion, and washing. ${ }^{47}$

The problem of maintaining both rapid transport of fluids and high oxygen permeability was solved by the introduction of glucose-sensitive silicone hydrogel ( $\mathrm{SiHG}$ ) lenses. A typical monomer contains a long silicone backbone and carbon-containing reactive groups at the ends for polymerization [Fig. 3(a)]. The interior of SiHG lenses has a different structure than HEMA lens [Fig. 2(b)]. The SiHG contains separate regions, which are $100 \%$ silicone and regions, which are $100 \%$ water. ${ }^{48,49}$ An important feature of these SiHG lenses is that these regions are organized in a unique manner so that the water channels and silicone-rich regions provide continuous pathways across the lens [Fig. 2(b)]. This spatial configuration is called an interpenetrating polymer network (IPN). The water channels provide tear fluid transport, and the silicone regions provide high oxygen transport [Fig. 2(b)], which can be higher than from an equivalent thickness of pure water [Fig. 3(b)]. The existence of IPN in SiHG lenses implies that there are interfaces between the low polarity silicone and the high polarity water channels. We recognized these regions could be used to bind amphipathic molecules at the interface. We further reasoned that previously known Glu-SFs, which work in water, ${ }^{36,37,43-46}$ could be modified with a hydrophobic moiety for binding to the lens while keeping the Glu-SF moiety in the aqueous phase and, hence, exposed to tear fluid. Subsequently, in this report, we first studied the IPN properties of $\mathrm{SiHG}$ contact lens using a polarity-sensitive probe Prodan followed by a Glu-SF that provides a reversible glucose response with long-term stability. Additionally, our recognition of the importance of IPNs provides a rational path to the design of other Glu-SFs. The introduction of this Glu-CL can be facilitated by the rapid evolution of consumer electronics, such as CMOS cameras, and methods to track the iris even if an eye is moving. It is difficult to predict the exact design of the device, which will detect emission from 
the Glu-CLs, but our approach can be adapted with a variety of optical detection configurations. It appears likely that Glu-CLs will contain multiple locations that provide known emission intensities for calibration and, thus, be self-calibrating.

\section{Experimental Methods}

\subsection{Materials}

The molecular structures of the fluorophores, Prodan, Quin, and Quin-C18, used for this study are shown in Fig. 4. The polaritysensitive probe Prodan was obtained from Sigma-Aldrich and employed as received. Quin was prepared using a previously described procedure by us. ${ }^{36,37}$ Quin-C18 synthesis involves a two-step procedure as shown schematically in Fig. 4(c). At first, 6-hydroxyquinoline was treated with 1-bromooctane in isopropyl alcohol in the presence of $\mathrm{NaOH}$ at $100^{\circ} \mathrm{C}$ for $6 \mathrm{~h}$ to obtain 6-octyloxyquinoline. Then, column purified 6-octyloxyquinoline was reacted with 2-(bromomethyl)phenylboronic acid in acetonitrile at room temperature for $12 \mathrm{~h}$ to obtain Quin-C18. The final product was recrystallized from hot acetonitrile and used for this study. The SiHG and HG contact lenses, used in this report are listed in Table 1, were obtained from commercial sources. These include three SiHG-type lenses [Lotrafilcon B (Optix Aqua), Comfilcon A (Biofinity), and Stenfilcon A (Aspire)] and one HEMA-type HG lens [Nelfilcon A (Dailies)] as a control to test our concept of localizing the Glu-SF at a silicone-water interface. The fluorophores were labeled by soaking the contact lenses in methanolic probe solutions for $1 \mathrm{~h}$ followed by washing them with water and buffer solutions for several times to eliminate the unbound probe from the contact lens. The dye-doped lenses were stored in water until they were used for the measurements. For probe (a)<smiles>CCC(=O)c1ccc2cc(N(C)C)ccc2c1</smiles>

(c)

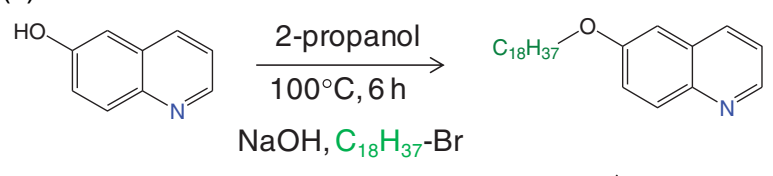

Fig. 4 Molecular structure of (a) Prodan, (b) Quin, and (c) Quin C18. (c) Shows the Quin-C18 synthetic scheme.
Table 1 Selected silicone $H G$ and $H G$ contact lenses for this study.

\begin{tabular}{lcccc} 
Polymer & Trade name & Manufacturer & $\begin{array}{c}\text { Water } \\
\text { content (\%) }\end{array}$ & DK \\
\hline Lotrafilcon B (SiHG) & Optix Aqua & CIBA Vision & 33 & 138 \\
Comfilcon A (SiHG) & Biofinity & Cooper Vision & 48 & 128 \\
Stenfilcon A (SiHG) & Aspire & Cooper Vision & 54 & 80 \\
Nelifilcon A (HG) & Dailies & CIBA Vision & 69 & 26 \\
\hline
\end{tabular}

washing-out experiments, the probe-doped contact lenses were taken in a scintillation tube with a buffer inlet and outlet accessory and subjected to $10 \mathrm{~min}$ of continuous buffer flow. The process is repeated multiple times before using the contact lens for the spectral measurements.

\subsection{Fluorescence Measurements}

Steady-state and time-resolved fluorescence measurements were performed using a specially designed holder that gently grips the lens without significant distortion, which is inserted into a $2.5-\times 0.8-\mathrm{cm}$ cuvette and typically at an angle near $60^{\circ}$ to avoid direct reflection off the surfaces toward the detection channel (Fig. 5). ${ }^{36,37}$ The solution surrounding the lens is easily changed to modify the glucose or fructose concentration. Fructose shows higher affinity to monoboronic acid probes and being considered for this study to know its binding trend in the contacts lens environment. Emission spectra and time-resolved decays were recorded with a FluoTime 300 instrument from PicoQuant. The amount of light incident on the contact lens and eye will be less than the intensity of bright sunlight. For example, the data shown in Fig. 6 were obtained using incident intensity

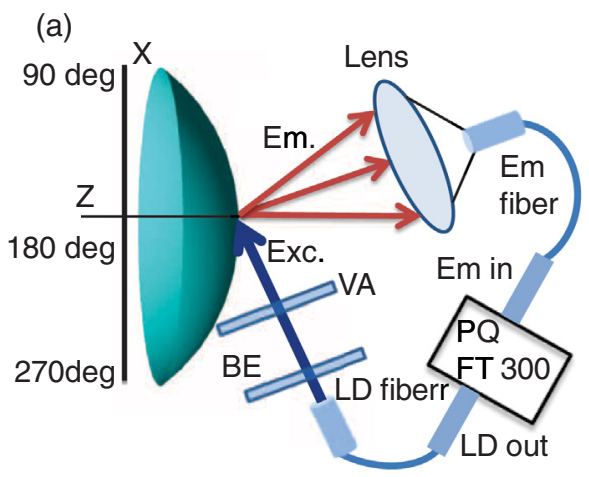

(b)

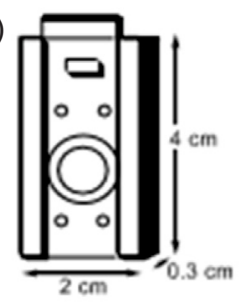

(c)

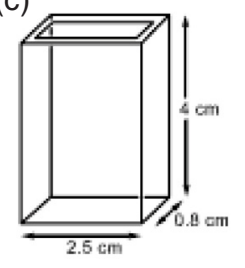

Fig. 5 (a) Optical configuration for measuring fluorescence from surfaces of contact lens. BE, beam expander; VA variable aperture; and LD, laser diode. (b) Device to hold contact lens. (c) A $2.5-\times 0.8-\mathrm{cm}$ cuvette. 
(a)
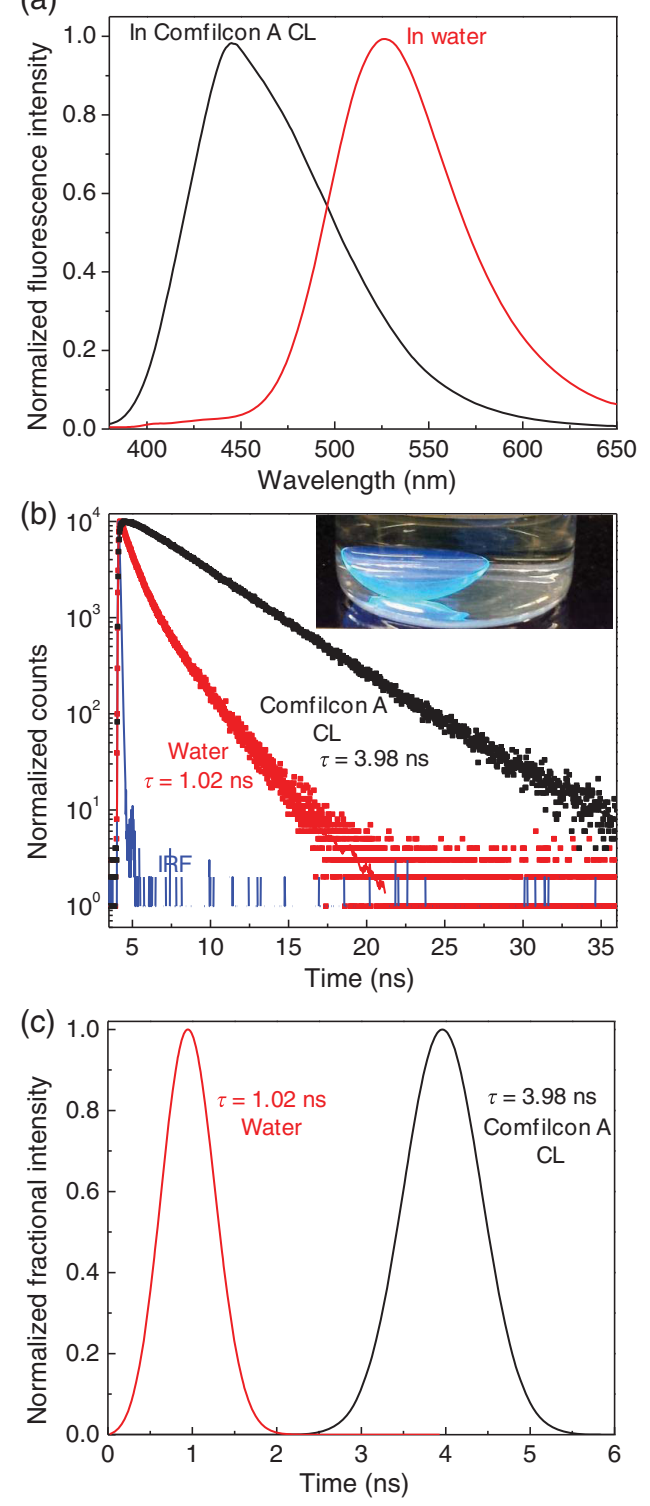

Fig. 6 (a) Emission spectra and (b) intensity decays of Prodan in a Comfilcon A contact lens or in water. Inset in (b) shows the labeled lens in a scintillation vial on a UV handlamp. (c) Lifetime distribution analysis of Prodan in water and Prodan-labeled Comfilcon A lens.

$<0.05 \mathrm{~mW} / \mathrm{mm}^{2}$. The average light intensity on the lens will be lower because the incident light can be turned off between measurements. The total solar irradiance reaching the earth from 350 to $700 \mathrm{~nm}$ is near $0.21 \mathrm{~mW} / \mathrm{mm}^{2},{ }^{50}$ which is about fourfold larger than the average excitation energy used for Fig. 6. The solar irradiance between 365 and $395 \mathrm{~nm}$ is near $0.04 \mathrm{~mW} / \mathrm{mm}^{2}$. For these reasons, we do not expect the incident light to be phototoxic to the cornea. Time-resolved decays were recorded at emission maxima of the probes. Fluorescence intensity and lifetime images were measured using an Alba laser scanning confocal microscope from ISS, Inc. The lifetime images were determined using frequency-domain phase angle measurements at a single modulation frequency of $40 \mathrm{MHz}$ as described in Ref. 51 but using a pixel-by-pixel basis by laser scanning.

\subsection{Fluorescence Lifetime Analysis}

Fluorescence intensity decays $I(t)$ are typically analyzed with the multiexponential model

$\mathrm{I}(\mathrm{t})=\sum_{i=1}^{n} \alpha_{i} \exp \left(-\frac{t}{\tau_{i}}\right)$,

where $\tau_{i}$ are the individual decay times and $\alpha_{i}$ are the amplitudes of each component at $\tau=0$, and $\Sigma \alpha_{i}=1.0 .^{52,53}$ The values of $\alpha_{i}$ and $\tau_{i}$ are determined using nonlinear least-squares (NLLS) analysis. The fractional contribution of each component to the total steady-state emission is proportional to the product of $\alpha_{i} \tau_{i}$. The multiexponential model makes the implicit assumption that fluorophores in the sample exist in discrete populations with specific decay times. In these initial studies of fluorophores in contact lenses, there is no reason to assume unique populations. Hence, the time-resolved decays were also analyzed using the lifetime distribution mode ${ }^{54,55}$ that allows continuous changes in the $\alpha$ and $\tau$ values. For this model, the intensity decay is represented by

$I(t)=\int_{\tau=0}^{\infty} \alpha(\tau) e^{-t / \tau} \mathrm{d} \tau$,

where $\int \alpha(\tau) \mathrm{d} \tau=1.0$. To decrease the number of variables in the NLLS analysis, we constrained the $\alpha(\tau)$ distribution to be a sum of Gaussian distributions. ${ }^{55}$ The results of this analysis are presented as the fractional contribution to the total intensity that is the product of the amplitude $\alpha$ multiplied by the lifetime $\tau$, that is, $\alpha\left(\tau_{i}\right)$. This mode of presentation provides results in an intuitive description of the overall intensity decays.

\section{Results and Discussion}

\subsection{Fluorophore Binding to an SiHG Contact Lens}

When we began these studies, we found no report on fluorophores bound to SiHG lenses. Therefore, it was necessary to confirm that the nonpolar silicone regions were accessible to molecules from the aqueous phase, and contact lenses contained hydrophobic regions that would bind nonpolar molecules. We selected the uncharged fluorophore Prodan that is known to be highly sensitive to local polarity. ${ }^{56}$ We incubated a Comfilcon A contact lens in a solution containing a low concentration of Prodan for $1 \mathrm{~h}$ as mentioned in Sec. 2. The lens was then washed in water for several hours to remove weakly bound Prodan. This procedure resulted in a highly fluorescent lens that can be seen near a UV headlamp in a scintillation vial [Fig. 6(b), inset]. The Prodan emission was strongly shifted toward shorter wavelengths as compared to water. This peak emission wavelength near $445 \mathrm{~nm}$ and the narrow width of the emission spectrum indicate that the Prodan is located in regions of low polarity. The homogeneity of this environment is confirmed by the time-dependent intensity decay of the probe in the lens, which is a single exponential with a decay time of $3.98 \mathrm{~ns}$. In contrast, Prodan in water displays a redshifted emission with band maximum of $540 \mathrm{~nm}$ and a shorter lifetime near 1.0 ns (Fig. 6). Further insights on Prodan location in contact lens were obtained from the lifetime distribution analysis of the intensity decays [Fig. 6(c)]. The decay times from Prodan within a Comfilcon A contact lens were found to be single exponential and have relatively wider lifetime distributions as 

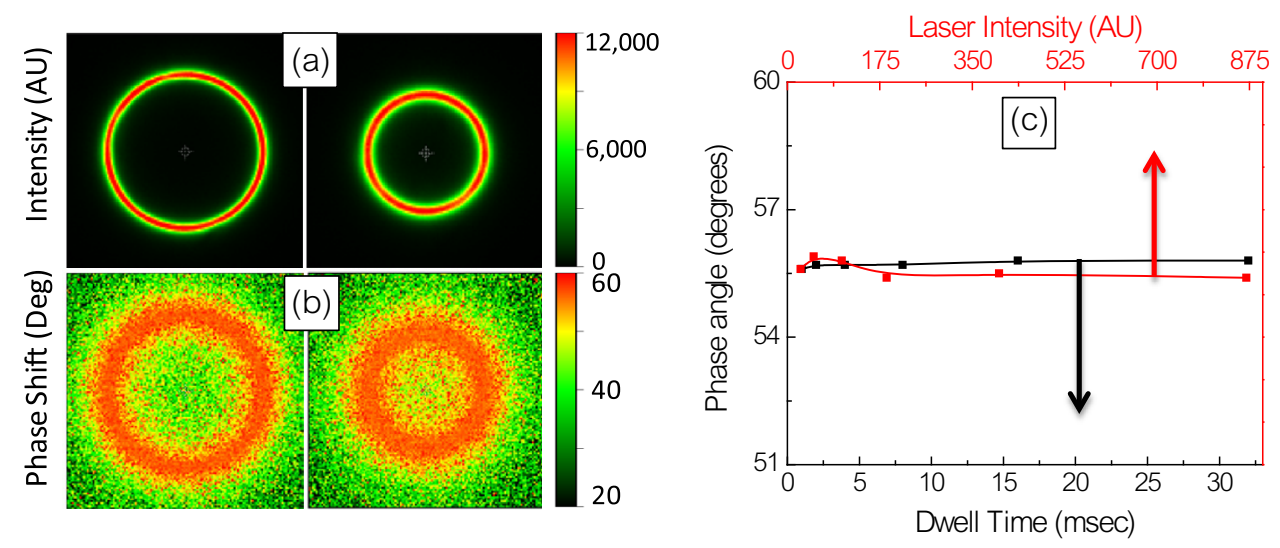

Fig. 7 (a) Effect of dwell time on accumulated intensity and phase angle, (b) effect of incident intensity on phase angle, and (c) summary plot for range of dwell time and laser intensity.

compared to that observed in water. The wider lifetime distribution in lens indicates relatively more, obvious, heterogenic environment in contact lens. More importantly, the absence of shorter decay time components in the Prodan lifetime distributions in contact lens represents the probe presence is insignificant in the polar aqueous regions of the contact lens. These results demonstrate two important attributes: (1) the hydrophobic molecules can readily diffuse into the SiHG (Comfilcon A) lens and (2) the existence of nonpolar regions in SiHG lenses.

The location of Prodan in the contact lens was further examined using laser scanning confocal fluorescence microscopy. The images obtained at different locations along the $Z$-axis show contact lens cross sections with different radii, which are consistent with confocal detection of the curved surface in an $X-Y$ plane. The absence of signal outside the lens indicates no detectable fluorophore is present in the surrounding solution [Fig. 7(a)]. Figure 7(b) shows the phase angle images of the lens. The uniform color indicates the lifetime is essentially similar in all regions of the lens. The phase angle is consistent with the 3.98-ns decay time as shown in Fig. 6. The measured phase angles are essentially independent of laser intensity or measurement times over a wide range of values [Fig. 7(c)]. This is an important result because fluorescence measurements, which are independent of the total intensity, will be necessary for glucose concentration self-testing where the optical geometry is uncertain and where ambient light is present.

\subsection{Testing of Glu-SF in HG Contact Lenses}

In our previous publications, we tested a large number of Glu-SFs that were responsive to glucose in buffer solutions. ${ }^{36,37}$ With one exception, these Glu-SFs did not respond to glucose when bound to HEMA-type contact lenses. We tested this one responsive probe, which we call Quin, in a Comfilcon A SiHG contact lens. Before that, we tested Quin response to glucose in buffer, and a $50 \%$ decrease in intensity in the presence of 100$\mathrm{mM}$ glucose was observed [Fig. 8(a)]. Quin lifetime in buffer was a single exponential and remained unchanged at $22 \mathrm{~ns}$ upon addition of glucose [Fig. 8(b)]. This is further corroborated with the lifetime distribution analysis, in which Quin shows single narrow lifetime distributions at 22 ns (data not shown). However, Quin is not suitable for a glucose-sensing contact lens because it is easily washed out of the SiHG lens [Fig. 9(a)] and would be removed from the contact lens by the continual replacement of tear fluid covering the eye,
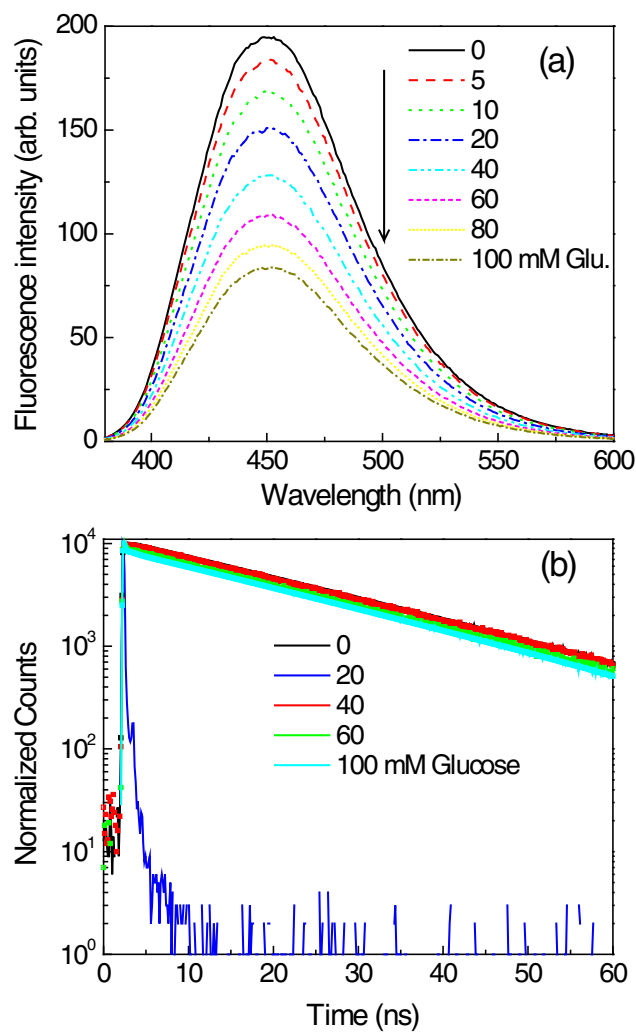

Fig. 8 Effect of glucose (a) on emission and (b) on emission intensity decays of Quin in $\mathrm{pH} 7.2$ phosphate buffer.

which occurs about every 10 min. ${ }^{57}$ This is because Quin is a hydrophilic molecule, readily soluble in water (aqueous solvents), and has no long C18 alkyl chain to bind with the contact lens interior regions.

To prevent probe leaching, it needs to be tethered to the contact lens either physically, such as in this study, or chemically with a covalent linkage. We choose to use physical adsorption of probe to minimize the complexity in modifying and re-establishing the characteristics of the lenses. For this reason, a derivative of Quin was synthesized with a C18 hydrophobic side chain [Fig. 4(c)]. The probe Quin-C18 could not be removed from a Comfilcon A ( $\mathrm{SiHG})$ lens even after extensive washing, using the procedure mentioned in Sec. 2 [Fig. 9(b)]. A thoroughly washed Quin-C18-doped lens displayed a bright blue 

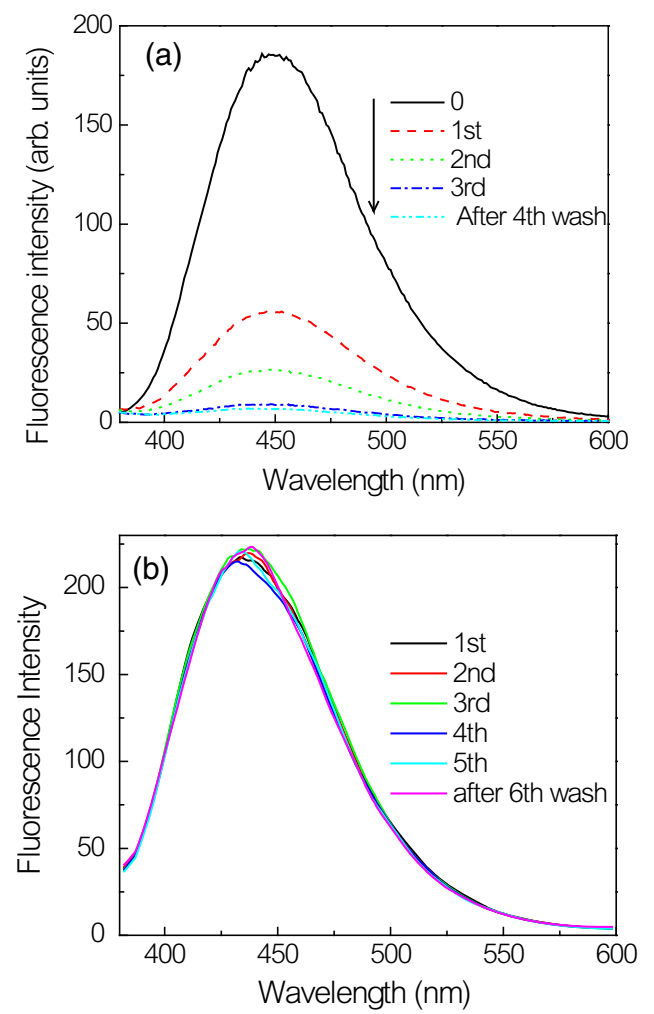

(c)

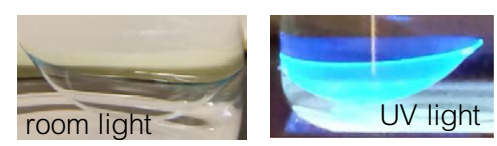

Fig. 9 Emission spectra of (a) Quin and (b) Quin-C18 in a Comfilcon A contact lens with repeated washings. (c) Photograph of Quin-C18doped Comfilcon A contact lens taken without an emission filter.

fluorescence that could be easily seen in room light [Fig. 9(c)]. Subsequently, we tested Quin-C18 response to glucose when doped in three different SiHG contact lenses (Fig. 10). Quin$\mathrm{C} 18$ emission intensity was reduced consistently with increasing concentrations of glucose. However, Quin-C18 did not respond to glucose when bound to, or in the presence of, a nonsilicone HG lens (Dailies in Fig. 10). The strong binding ability and glucose response of Quin-C18 in SiHG lenses, but not HG lenses, confirmed the need for a nonpolar-to-polar interface region, schematically shown in Fig. 2(b), where the hydrophobic C18 side chain can help binding the Quin-C18 to contact lens and hydrophilic boronic acid moiety of the probe is positioned in the water channels of lens and, thus, able to show response to glucose. Similar to Quin, the intensity decays of Quin-C18 did not change in response to glucose binding [Fig. 11(a)]. However, the lifetime of Quin-C18 bound to the lens is relatively shorter (with amplitude weighted average lifetime of $7 \mathrm{~ns}$ ), which can only be best-fitted with multiexponential decay parameters. As per our understanding, Quin-C18 is not an environment-sensitive probe and we are puzzled to notice the short lifetime for the probe in contact lens as compared to that in pure solvent, such as methanol. The lifetime distributions of Quin-C18 in methanol and in an SiHG lens are shown in Fig. 11(b). As can be seen from the figure, the lifetime distribution of Quin-C18 in methanol has single Gaussian peak with a lifetime at $25.5 \mathrm{~ns}$ and that for Quin-C18 in contact lens has
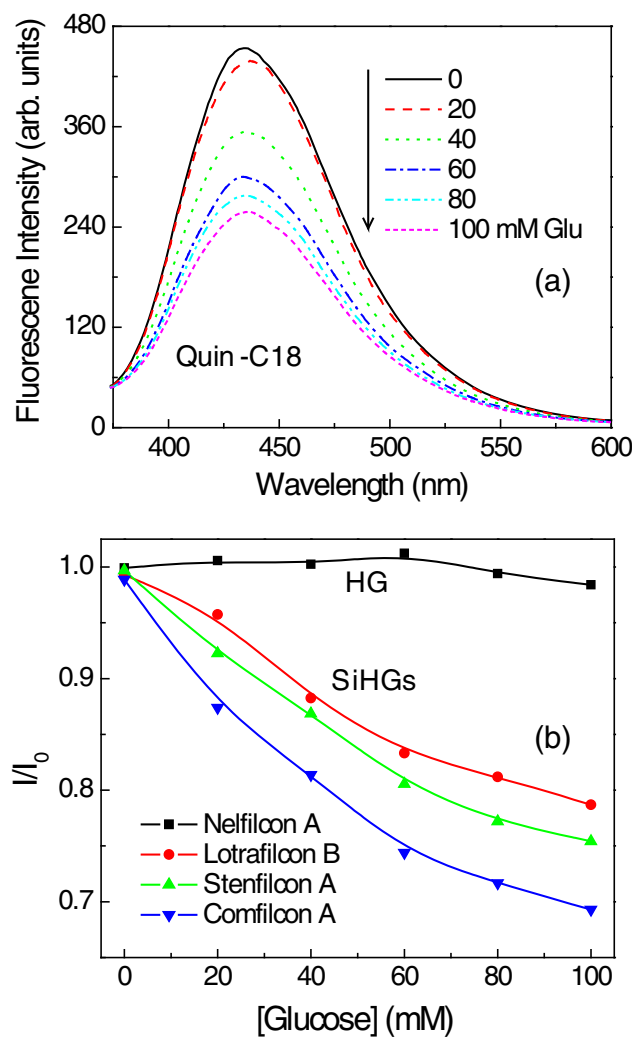

Fig. 10 (a) Glucose-dependent emission spectra of Quin-C18 within a Comfilcon A (Biofinity) SiHG lens. (b) Normalized emission intensities of Quin-C18 within a Nelfilcon A (HG) lens and that in three SiHG lenses: Comfilcon A (Biofinity), Stenfilcon A (Aspire 1day), and Lotrafilcon B (Optix Aqua) lenses.

two maxima centered at about 2.6 and $15.9 \mathrm{~ns}$. Moreover, the latter distribution is slightly broad indicating the probe within the lens is in more heterogeneous environment than that in methanol. The refractive index at interface may affect the lifetime of the probe. However, the behavior we noticed with Quin-C18 is different from the other probes used within the lens. For example, lifetime behavior of Prodan in contact lens (Figs. 6 and 7) shows no refractive index effect. We noticed insignificant refractive index effect on NBD-C18 in SiHG lens. ${ }^{58}$ Owing to the polarity-insensitive nature of Quin-C18, additional experimentation is required to understand the discrepancies in the lifetime distribution of Quin-C18 in solution and in contact lens.

For use in continuous analyte monitoring, the fluorophores must have long-term stability and a reversible response to analyte. Glucose-sensing fluorophores developed using boronic acid are known to show reversible response to glucose. This is because boronic acid binding with glucose is reversible (Fig. 1). Figure 12 shows the Quin-C18-doped SiHG lenses response to glucose or fructose. The emission intensities were measured at 10-min intervals with continuous flow of buffer or buffer with varied concentrations of glucose or fructose. Initial five points in the response curve with continuous flow of buffer indicate Quin-C18 is firmly bound to the SiHGs. Subsequently, consistent decrease in emission intensity was noticed with increasing concentrations of glucose in buffer, and similar response from all three SiHGs used in this study can be seen. The emission intensity was completely recovered by purging buffer alone. 

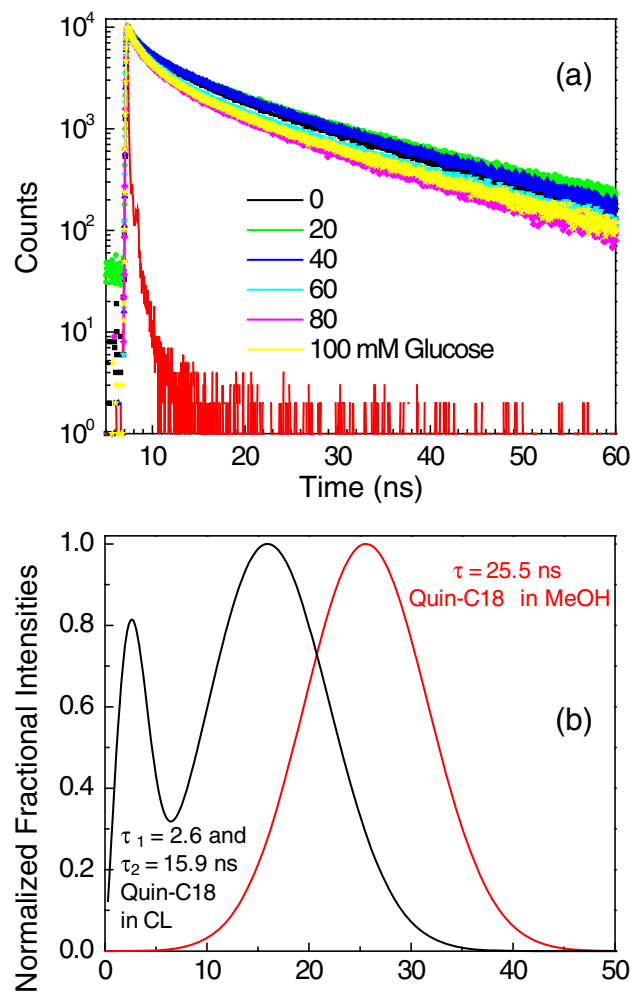

Fig. 11 (a) Effect of glucose on the time-dependent decays of QuinC18 in Comfilcon A lens. (b) Lifetime distribution of Quin-C18 in methanol and Quin-C18-labeled Comfilcon A lens.

Then, we tested the same lenses, purged with buffer for $50 \mathrm{~min}$, response to fructose, which is known to show higher affinity to monoboronic acids, by purging buffer containing fructose. As expected, all three lenses show better response to fructose over glucose, and moreover, once again the emission intensity can be completely recovered by purging with the buffer alone. Almost no response either with glucose or fructose was noticed when the probe is bound to the HG lens (Dailisis). The present data indicate that the Quin-C18 lenses show consistent reversible response to monosaccharides. Then, to test the shelf life of the lenses, we measured the glucose and fructose response from the lenses used for Fig. 12(a), which were stored in water for three months. The water-stored lenses show similar response toward glucose or fructose to that of the freshly doped lenses. Also, the HG lens once again did not respond to glucose (Fig. 12). This result suggests that a probe, such as Quin-C18, can be used in extended wear contact lenses.

The results for Quin and Quin-C18 were obtained using high glucose concentrations up to $100 \mathrm{mM}$, suitable for blood glucose range. This is because glucose binds weakly to these probes with single boronic acid residue. The glucose concentration in tears is thought to be about sevenfold lower than in blood (5 mM) so that a Glu-SF must be sensitive to glucose concentrations below $1 \mathrm{mM}$. This can be accomplished using Glu-SFs that contain two boronic acid groups. Diboronic acid Glu-SFs are known to display binding constants near $0.5 \mathrm{mM}^{46,59}$ Currently, we are designing a diboronic acid probe that is suitable for the low glucose concentrations and the lenses with this new probe may be appropriate for glucose monitoring using tears. However, the presented results emphasize the concept of using ampipathic sensors within SiHG lenses for
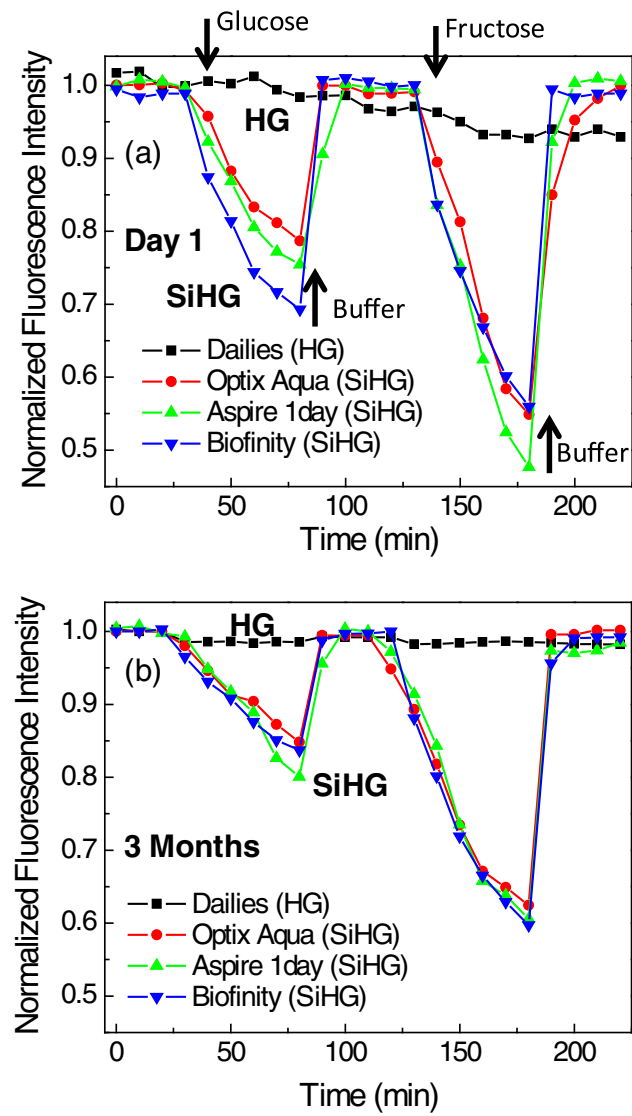

Fig. 12 (a) Response of Quin-C18 to glucose or fructose within different $\mathrm{SiHG}$ and in a HG in $\mathrm{pH} 7.2$ phosphate buffer. Incrementing glucose concentrations (from 0 to 100 with $20 \mathrm{mM}$ interval) and 0, 1, 2, 4, 7 , and $10 \mathrm{mM}$ of fructose were used before purging the lenses with final buffer. Bioaffinity, Aspire 1day, and OptixAqua are SiGHs and Dailies is a HG contact lens. (b) Similar response to the glucose or fructose from Quin-C18-doped lenses stored in water for 3 months.

continuous, noninvasive monitoring tear glucose is realistic in near future.

\section{Conclusion}

Herein, for the first time, we have described an approach to measure possible tear glucose with taking advantage of the internal structure of present SiHG lenses. We anticipate that many known Glu-SFs, which show response to glucose in water/buffer conditions and not in contact lenses, can now be made suitable for contact lens based sensing by the presented approach. These Glu-CLs can provide immediate health benefits to individuals with diabetes who already wear contact lenses. The use of these lenses is likely to find additional uses in juveniles or individuals who are unable or unwilling to perform frequent BGST. Our concept of using the interfacial region of SiHG lenses can also be used with fluorescent probes that are sensitive to many other analytes. In fact, we tested the possibility of the approach presented in this paper for $\mathrm{pH}$ and chloride, and results show that the modified contact lens could be useful for the diagnosis of dry eye disease. ${ }^{58}$

\section{Disclosures}

The authors have no relevant financial interests in this article and no potential conflicts of interest to disclose. 


\section{Acknowledgments}

This work was supported by Grants from the National Institutes of Health (Nos. GM125976, GM107986, EB006521, EB018959, and OD019975). The authors thank Dr. Liangfu $\mathrm{Zu}$ and Dr. Douguo Zhang for assistance with the artwork, Dr. Henryk Szmacinski for assistance with the FLIM measurements, and Dr. Julie Rosen and Ms. Mary Rosenfeld for critical reviewing and editing.

\section{References}

1. WHO, "Global report on diabetes," pp. 1-86 (2016), http://apps.who. int/iris/bitstream/10665/204871/1/9789241565257_eng.pdf.

2. M. E. Tucker, "IDF Atlas: about 415 million adults worldwide have diabetes," in Int. Diabetes Federation (IDF) 2015 World Congress (2017), http:www.medscape.com/viewarticle/866296.

3. I. B. Hirsch and D. E. DeWitt, "Outpatient insulin therapy in type 1 and type 2 diabetes mellitus: scientific review," JAMA 289, 2264-2254 (2003).

4. J. J. Duby et al., "Diabetic neuropathy: an intensive review," Am. J. Health Syst. Pharm. 61, 160-173 (2004).

5. S. Boudina and E. D. Abel, "Diabetic cardiomyopathy revisited," Circulation 115, 3213-3223 (2007).

6. A. Correa et al., "Diabetes mellitus and birth defects," Am. J. Obstet. Gynecol. 199, 237.e1-237.e9 (2008).

7. E. Stefansson et al., "Screening and prevention of diabetic blindness," Acta Ophthalmol. 78, 374-385 (2000).

8. E. A. Reece, "The fetal and maternal consequences of gestational diabetes mellitus," J. Matern.-Fetal Neonat. Med. 23, 199-203 (2010).

9. M. S. Ola et al., "Recent advances in understanding the biochemical and molecular mechanism of diabetic retinopathy," J. Diabetes Complications 26, 56-64 (2012).

10. A. G. A. Aggidis, J. D. Newman, and G. A. Aggidis, "Investigating pipeline and state of the art blood glucose biosensors to formulate next steps," Biosens. Bioelectron. 74, 243-262 (2015).

11. J. C. Pickup et al., "In vivo glucose monitoring: the clinical reality and the promise," Biosens. Bioelectron. 20, 1897-1902 (2005).

12. C. E. F. do Amaral and B. Wolf, "Current development in non-invasive glucose monitoring," Med. Eng. Phys. 30, 541-549 (2008).

13. J. Wang, "Glucose biosensors: 40 years of advances and challenges," Electroanalysis 13(12), 983-988 (2001).

14. X. Guo, A. Mandelis, and B. Zinman, "Noninvasive glucose detection in human skin using wavelength modulated differential laser photothermal radiometry," Biomed. Opt. Express 3(11), 3012-3021 (2012).

15. A. J. Bandodkar et al., "Tattoo-based noninvasive glucose monitoring: a proof-of-concept study," Anal. Chem. 87, 394-398 (2015).

16. M. Mortellaro and A. DeHennis, "Performance characterization of an abiotic and fluorescent-based continuous glucose monitoring system in patients with type 1 diabetes," Biosens. Bioelectron. 61, 227-231 (2014).

17. G. Zenkl and I. Klimant, "Fluorescent acrylamide nanoparticles for boronic acid based sugar sensing-from probes to sensors," Microchim. Acta 166, 123-131 (2009).

18. H. Shibata et al., "Injectable hydrogel microbeads for fluorescence based in vivo continuous glucose monitoring," Proc. Natl. Acad. Sci. U. S. A. 107(42), 17894-17898 (2010).

19. H. Lee et al., "A graphene-based electrochemical device with thermoresponsive microneedles for diabetes monitoring and therapy," Nat. Nanotechnol. 11, 566-572 (2016).

20. J. Wang, "In vivo glucose monitoring: towards 'sense and act' feedbackloop individualized medical systems," Talanta 75, 636-641 (2008).

21. B. C. Crane et al., "The development of a continuous intravascular glucose monitoring sensors," J. Diabetes Sci. Technol. 9(4), 751-761 (2015).

22. D. Rodbard, "Continuous glucose monitoring: a review of successes, challenges, and opportunities," Diabetes Technol. Ther. 18(2), S2-S3 (2016).

23. L. Heinemann and J. H. DeVries, "Reimbursement for continuous glucose monitoring," Diabetes Technol. Ther. 18(2), S2-48 (2016).

24. N. S. Oliver et al., "Glucose sensors: a review of current and emerging technology," Diabetic Med. 26, 197-210 (2009).
25. I. Amato, "Race quickens for non-stick blood monitoring technology," Science 258, 892-893 (1992).

26. V. L. Alexeev et al., "High ionic strength glucose-sensing photonic crystal," Anal. Chem. 75, 2316-2323 (2003).

27. B. Rabinovitch, W. F. March, and R. L. Adams, "Non-invasive glucose monitoring of the aqueous humor of the eye: part I. Measurement of very small optical rotations," Diabetes Care 5(3), 254-258 (1982).

28. N.-T. Yu et al., "Development of a noninvasive diabetes screening device using the ratio of fluorescence to Rayleigh scattered light," J. Biomed. Opt. 1(3), 280-288 (1996).

29. N. Saiga, C. Hamada, and J. Ikeda, "Near infrared spectroscopy assessment of the glucose solution processed by ultrasound cavitation," Ultrasonics 44, e101-e104 (2006).

30. M. J. Tierney et al., "Clinical evaluation of the GlucoWatch ${ }^{\circledR}$ biographer: a continual, non-invasive glucose monitor for patients with diabetes," Biosens. Bioelectron. 16, 621-629 (2001).

31. S. Y. Rhee et al., "Clinical experience of an iontrophoresis-based glucose monitoring system," J. Korean Med. Sci. 22, 70-73 (2007).

32. S. Gebhardt et al., "Glucose sensing in transdermal body fluid collected under continuous vacuum pressure via microspores in the stratum corneum," Diabetes Technol. Ther. 3, 81-90 (2001).

33. J. T. LaBelle et al., "Self-monitoring of tear glucose: the development of a tear based glucose sensor as an alternative to self-monitoring of blood glucose," Chem. Commun. 52, 9197-9204 (2016).

34. J. T. Baca, D. N. Finegold, and S. A. Asher, "Tear glucose analysis for the noninvasive detection and monitoring of diabetes mellitus," Ocul. Surf. 5(4), 280-293 (2007).

35. S. Iguchi et al., "Biomedical soft contact-lens sensor for in situ ocular biomonitoring of tear contents," Biomed. Microdevices 9, 603-609 (2007).

36. R. Badugu, J. R. Lakowicz, and C. D. Geddes, "Ophthalmic glucose monitoring using disposable contact lenses," Rev. Fluoresc. 2005, 363-397 (2005).

37. R. Badugu, J. R. Lakowicz, and C. D. Geddes, "Fluorescence sensors for monosaccharides based on the 6-methylquinolinium nucleus and boronic acid moiety: potential application to ophthalmic diagnostics," Talanta 65, 762-768 (2005).

38. http://www.visionaware.org/blog/visionaware-blog/googles-prototypesmart-contact-lens-measuring-blood-glucose-levels-for-people-withdiabetes.

39. H. Yao et al., "A contact lens with integrated telecommunication circuit and sensors for wireless and continuous tear glucose monitoring," J. Micromech. Microeng. 22, 075007 (2012).

40. P. L. Rakow, "Silicone hydrogels, what, why, and how," Eyewitness Fourth Quarter, pp. 21-25, CL Society of America, http://www.clsa. info/PDF/4Q_06_CUP230.pdf (29 January 2008).

41. W. Townsend, "How daily disposable lenses can address contact lens dryness," Contact Lens Spectrum, Special Edition, p. 12 (2013), www.cispectrum.com.

42. P. B. Morgan and N. Efron, "A decade of contact lens prescribing trends in the United Kingdom (1996-2005)," Contact Lens Anterior Eye 29, 59-68 (2006).

43. S. Arimori et al., "A modular fluorescence intramolecular energy transfer saccharide sensor," Org. Lett. 4(24), 4249-4251 (2002).

44. H. Fang, G. Kaur, and B. Wang, "Progress in boronic acid-based fluorescent glucose sensors," J. Fluoresc. 14(5), 481-489 (2004).

45. M. S. Steiner, A. Duerkop, and O. S. Wolfbeis, "Optical methods for sensing glucose," Chem. Soc. Rev. 40, 4805-4839 (2011).

46. T. D. James et al., "Novel saccharide-photoinduced electron transfer sensors based on the interaction of boronic acid and amine," J. Am. Chem. Soc. 117, 8982-8987 (1995).

47. P. C. Nicolson and J. Vogt, "Soft contact lens polymers: an evolution," Biomaterials 22, 3273-3283 (2001).

48. J. P. Kennedy and U. Subramanyam, "Polymer networks, producing crosslinking polyvinyl alcohol-polysiloxanes, and products and films made from networks," US 20100305231 A1 20101202 (2010).

49. S.-H. Chu and H.-H. Cheng, "3D network structured silicon containing prepolymer and method for its manufacture," US 20150252194 A1 20150910 (2015).

50. W. W. Gregg and K. L. Carder, "A simple spectral solar irradiance model for cloudless maritime atmospheres," Limnol. Oceanogr. 35(8), 1657-1675 (1990). 
51. H. Szmacinski and J. R. Lakowicz, "Lifetime-based sensing," in Topics in Fluorescence Spectroscopy, J. R. Lakowicz, Ed., pp. 295-334, Plenum Press, New York (2006).

52. A. Grinvald and I. Z. Steinberg, "On the analysis of fluorescence decay kinetics by the method of least-squares," Anal. Biochem. 59, 583-598 (1974).

53. D. V. O'Connor and D. Phillips, Time-Correlated Single-Photon Counting, p. 288, Academic Press, New York (1984).

54. J. R. Alcala, E. Gratton, and F. G. Prendergast, "Fluorescence lifetime distributions in proteins," Biophys. J. 51, 597-604 (1987).

55. J. R. Lakowicz et al., "Analysis of fluorescence decay kinetics measured in the frequency-domain using distribution of decay times," Biophys. Chem. 28, 35-50 (1987).

56. R. Hutterer and M. Hof, "Probing ethanol-induced phospholipid phase transitions by the polarity sensitive fluorescence probes Prodan and Patman," Z. Phys. Chem. 216, 333-346 (2002).

57. P.-T. Yeh, R. Casey, and B. J. Glasgow, "A novel fluorescent lipid probe for dry eye: retrieval by tear lipocalin in humans," Invest. Ophthalmol. Visual Sci. 54, 1398-1410 (2013).

58. R. Badugu et al., "Contact lens to measure individual ion concentrations in tears and applications to dry eye disease," Anal. Biochem. 542, 84-94 (2018).

59. V. Karnati et al., "A glucose-sensitive fluorescence sensor based on boronic acid diol recognition," Bioorg. Med. Chem. Lett. 12, 3373-3377 (2002).
Ramachandram Badugu is an assistant professor at the Center for Fluorescence Spectroscopy, Department of Biochemistry and Molecular Biology, University of Maryland, Baltimore. His research interests are development of methodologies for fluorescence sensing, fluorescence signal amplification, and/or directional emission using plasmonic, photonic, or hybrid plasmonic-photonic structures.

Edward Albert Reece is an executive vice president for medical affairs at the University of Maryland, the dean of the School of Medicine, and a professor in the Departments of Obstetrics, Gynecology and Reproductive Sciences; Internal Medicine, and Biochemistry and Molecular Biology. His research interests are studying the biologic/molecular causes and consequences of diabetesinduced birth defects.

Joseph R. Lakowicz is a professor and a director of the Center for Fluorescence Spectroscopy, Department of Biochemistry and Molecular Biology, University of Maryland, Baltimore. His research is focused on advancing the field of fluorescence spectroscopy, which includes fluorescence sensing and development of plasmoncontrolled fluorescence. 